\title{
Mislabelled hospital laboratory specimen-a risk assessment perspective
}

\author{
Anne Rowshan ${ }^{1}$, Hooman Rowshan ${ }^{2}$ \\ ${ }^{1}$ Hospital for Sick Children, Toronto, Canada, York University Department of Nursing, Toronto, Canada \\ ${ }^{2}$ College of Pharmacy, University of Florida, Gainesville, Florida, USA
}

\section{Email address:}

Anne.Vongprachanch-Rowshan@sickkids.ca (A. Rowshan), hrowshan@fastmail.us (H. Rowshan)

\section{To cite this article:}

Anne Rowshan, Hooman Rowshan. Mislabelled Hospital Laboratory Specimen-A Risk Assessment Perspective. American Journal of Nursing Science. Vol. 3, No. 1, 2014, pp. 1-4. doi: 10.11648/j.ajns.20140301.11

\begin{abstract}
Mislabeling of laboratory samples has been found to be a high-risk issue in acute care hospitals. In cases where hospitals adopted a bar code tracking system to ensure proper identification of specimens moving from bedside to the laboratory, the error rates were reduced from 108 to just 8 . It is therefore concluded that adoption of bar code tracking system is an effective strategy for reducing error rate when sample specimen are collected at the bedside and transmitted to the laboratory. In this literature review, we propose to explore the use of bar code technology as and alternative method to reduce mislabeling of patients' specimen. Although the focus group assembled to explored the risk assessment issues surrounding the mislabeled labeled specimen did not, we believe based on the review of the existing literature that bar code technology is the only method that provide the most patient safety.
\end{abstract}

Keywords: Mislabeling of Laboratory Samples, Specimen Collection, Delta Checking, Preanalytic Errors, Analytic Errors, Laboratory Errors, Patient Impact, Physician and Nursing Responsibilities

\section{Introduction}

To ensure patient safety, all medical staff is expected to observe to a policy with respect to mislabeled and unlabeled specimens. A mislabeled specimen is one in which the name/medical record or patient identifiers on the specimen label do not match what is sent the laboratory. An unlabeled is one, which arrives at the laboratory with the without a specimen label providing required patient identifiers. The College of American Pathologists estimates 1 in 1,000 blood specimens have wrong labels with the wrong patient identifiers. This type of error potentially harms two parties - the patient whose blood was mislabeled and the patient who was wrongly linked to that specimen. Both patients may end up with incorrect diagnoses, harmful treatment, or treatment they do not need.

There are three common types of patient documentation errors: (1) discrepancy between the requisition label and the specimen label, (2) unlabeled specimens that include both completely unlabeled specimens and specimens with only one of the two required identifiers, and (3) completely misidentified specimens.

At the outset, it is important to use one standardized definition for mislabeled specimen. There are may terminology is in use in the literature but an institutional definition is always warranted and must be the first step in establishing a quality control program. The reason is obvious: this is because it improves the quality improvement, particularly if laboratories want to equate results.

In this article we propose two ways to reduce the incidents of mislabeled specimens: First we propose an institutional wide education focusing specifically on those aspects of workflow likely to result in errors. We propose supplementing institutional wide education with an electronic database to record cases in which mislabeling has occurred. The data collected over time in such a system maybe used for quantitative quality control studies on periodic basis. Finally, we propose the implementation of a bar code system in order to significantly reduce mislabeling errors. This latter proposal is discussed later and is support by current literature. 


\section{Current Practice at the Critical Care Unit of the Hospital for Sick Children}

Specimens are collected by the bedside nurse and transported via the pneumatic system to the on site hospital laboratory. An order for specimen collection is obtained from the physician and the bedside nurse prints the laboratory requisition using a common printer for the particular room. A double check for the correct patient identifying information is performed prior to collection by matching two patient identifiers on the requisition with the patient's armband. The bedside nurse collects the specimen and affixes the label to the specimen container with a unique ascension number. Once the specimen is collected, a second independent check is completed to ensure the specimen belongs to the correct patient. Following collection of the specimen, the specimen is packaged either with ice or without ice in biohazard bags and sent to the laboratory. Errors in mislabeled specimen have been found to include identification error whereby the patient's armband is not present on the patient or it is overlooked in the double check process because staff members too often believe their familiarity with a particular patient obviates the need for the patient identification verification. Another issue arises when the individual obtaining the specimen is not the same individual who affixes the label to the sample or transports the specimen to the pneumatic system. In our current system, several omissions along key steps may occur for various reasons increasing the likelihood for mislabeling of specimens.

\section{Focus Group Recommendations}

The Quality and Safety Group identified a number of points in the collection, processing and transportation of specimens that contributed to preanalytic errors. These include multiple people assisting with specimen collection and different people printing up the requisition and affixing the labels to the specimens. Other issues that came to light were printer problems that delayed in printing up requisitions, failure of the bedside nurse to double check the armband identification with the requisition, emergency situations in which staff were rushed, frequent blood sampling, and printing errors in which the wrong patient requisition is printed. The recommendation that resulted from these findings suggested that in order to minimize preanalytic errors, only one person, namely the patient's bedside nurse, should be involved in the entire collection, processing, labeling and transport of the specimens from start to finish. It would be ideal to have the bedside nurse complete this process; however, the critical care unit is unpredictable as patients' status changes without notice and when a child deteriorates, many hands are required for resuscitation. Hence there is still a great possibility for error in such chaotic situations.

\section{Literature Review}

Errors in laboratory services involve a number of problems, which may occur outside or inside the laboratory. [2] There are standard terminologies attributed to each type of error. Preanalytic errors are those that occur before the laboratory analyzes a specimen. The simplest example of preanalytic error is mislabeling error. In fact, specimen mislabeling accounts for the most cases of preanalytic errors. Much of the incorrect lab results, which physicians and nurses attribute to errors in the laboratory analytical procedures are actually the result of preanalytic problems occurring before the laboratory receives the specimen. Analytic errors are those occurring during the manual or automated specimen analysis in the laboratory. Post analytic errors occur after the wrapping up of the analytical procedure. A good illustration of post analytic error consist of misreporting results by oral, written, or electronic communication. [3,4,5].

Relative Studies are difficult to merge due to disparities in error recognition methods and intuitively defined definitions of what establishes error. Published error rates are likely inaccurate because of insufficient detection methods and reluctance by different facilities to share their error rates. However, peer-reviewed studies attribute approximately $0.05 \%$ overall error rates to specimen mislabeling [6].

In one recent report, investigators determined the most common reasons for rejection by laboratory of the submitted samples were improperly labeled specimen containers (14.46\%). [7] In 2012, Snydman, et.al, conducted a cross-sectional study of reported unfavorable laboratory results from 30 health organizations throughout the United States (January 1, 2000, to December 31, 2005). Those investigators found preanalytic laboratory adverse events were the most common $(81.1 \%)$; the most top 3 preanalytic laboratory events were specimen not labeled properly $(18.7 \%)$, specimen mislabeled (16.3\%), and improper collection (13.2\%). A small number of preanalytic $(0.08 \%)$ of laboratory adverse events resulted in permanent harm or death to the patients; $8 \%$ of preanalytic error resulted in temporary harm. Most adverse laboratory events (55\%), however, did not cause harm. The Snydman study concluded preanalytic laboratory errors constitute 1 out of 7 unfavorable quality events. The authors concluded "Laboratory errors often are caused by events that precede specimen arrival in the lab and should be preventable with a better labeling processes and education. Most laboratory errors do not lead to patient harm". [8].

Hill and colleagues from the department of emergency medicine at Johns Hopkins University conducted a study to measure the rate of reduction in specimen processing error after implementation of an electronic physician order entry system, which included a bar-coded labeling process. Of the 724,465 specimens collected pre-intervention, 3,007 $(0.42 \%)$ were recorded as errors as opposed to 379 errors $(0.11 \%)$ of 334,039 specimens collected post intervention, 
which represents a $74 \%$ relative and $0.31 \%$ absolute decrease ( $95 \%$ confidence interval $0.28 \%$ to $0.32 \%$ ). The proportion of institutional errors contributed by the ED was reduced from $20.4 \%$ to $11.4 \%$, a $44 \%$ relative and $9.0 \%$ absolute reduction (95\% confidence interval $7.7 \%$ to $10.3 \%$ ). A further improved analysis revealed the majority of continued errors occur when the physician order entry/bar-code system could not be used (e.g., blood bank or surgical pathology specimens). The authors concluded "combining an electronic physician order entry with barcoded patient verification and electronic documentation and information system-generated specimen labels which significantly reduce specimen errors, with a sizable influence on institutional specimen-related errors." At the same time, this study cautions that continued use of hand labeling specimen is inadvisable in institutional settings. Although some institutions may be inclined to bypass automated specimen processing protocol in favor of cost saving measures, findings of the study from Johns Hopkins states the cost savings of non-automated specimen processing systems remains unsubstantiated. [9].

The frequencies of wrong therapeutic intervention based solely on mislabeled specimen have been compared between US and European institutions. A University of Washington study concluded the rate of error among the US partakers in the study were comparable to those reported for most European countries. [10] Institutions who replaced a step in their work flow process by requiring manual preprinting of labels experienced labeling errors in about one quarter of specimens collected. However, this error rate was removed after the implementation of a bar code-based patient identification system. [11].

There are many ways in which specimen collections errors are discovered. Some are obvious while others not as readily apparent. [1] The caregiver may discover the errors, incident reports from staff inside or outside the laboratory, internal error checking audit protocols within the laboratory, or automated laboratory management reports. Mislabeling errors in particular are detected predominantly by two methods in most institutions. It should be noted that most studies support the notion that only about $15 \%$ of mislabeled specimens result in the release of a laboratory result based on wrong specimen. These are laboratory results that have escaped the internal laboratory audit protocol and give rise to an incorrect data being released to medical and nursing staff. That is to say that in $85 \%$ of cases where specimens have been mislabeled, the laboratory detects them before the specimen is run or results reported. [6].

The laboratory itself is also skilled at detecting mislabeled specimen. This process is known as "delta checking". This is an internal laboratory safeguard that is effective in preventing mislabeled specimen from being run and incorrect results being released to caregivers. In delta checking, a laboratory-automated system is used as a safeguard. This involves an automated comparison of the patient's current and previous lab values, and the comparison takes place within the laboratory information system. If the difference between the values ("delta") is greater than a predefined limit, the result is flagged, and a procedure is implemented to determine if an error has occurred. Delta checking procedures vary among different laboratories but usually involve repeating the test and investigating for misidentification of specimen label. [14].

The advantage of delta checking is it does not rely on human accuracy. But the system has its shortcomings: For delta checking to be effective, the automated system requires previous values. Moreover, delta checking is difficult to apply to results where large changes are expected in common clinical conditions (for example, troponin in myocardial infarction). In addition, delta limits are imperfect since they involve the inherent tradeoff between false positive and false negative error detection.

A few rigorous studies have correlated errors in specimen mislabeling with adverse patient outcomes. Based on this limited data, it is likely that $5 \%-10 \%$ of laboratory service errors have led to patient harm. When there have been adverse effects associated with patient outcomes, most have occurred in acute settings. [4,12,13].

According to Astion, there are different types of intervention that might be utilized to reduce institutional laboratory error rates. These interventions range from weak strategies to intermediate, and strong. Strong intervention is one, which requires institutional transition to an automated, barcode-based system where there is less reliance in human vigilance, and more dependence on technology. The examples of weak and intermediate intervention are those that rely on continuous training and retraining, including active observation of nursing staff involved in specimen collection and labeling. [16]

\section{Conclusions}

The strong intervention as postulated by Astion is more expensive and difficult to implement, but it is likely to be the only real robust solution to the pervasive institutional problem of specimen mislabeling. However in the long run, we are likely to realize a reasonable financial return on investment if the true costs of mislabeling could be determined. But as mentioned earlier, there is no data yet that truly establishes the true cost of mislabeling. Hence, the risk benefit analysis remains somewhat subjective.

\section{Competing Interests}

Authors state they have no conflict of interest.

The authors would like to acknowledge the contributions of the members of "Quality and Safety Committee" at the Hospital for Sick Children for their insight, which culminated in the production of this manuscript. 


\section{References}

[1] Brown, Judy E., Nancy Smith, and Beth R. Sherfy. "Decreasing mislabeled laboratory specimens using barcode technology and bedside printers." Journal of Nursing Care Quality 26.1 (2011): 13-21.

[2] Bonini P, Plebani M, Ceriotti F, Rubboli F. Errors in laboratory medicine. Clin Chem. 2002;48:691-698.

[3] Lapworth R, Teal TK. Laboratory blunders revisited. Ann Clin Biochem. 1994; 31:78-84.

[4] Plebani M, Carraro P. Mistakes in a stat laboratory: Types and frequency. Clin Chem

[5] Howanitz PJ. Errors in laboratory medicine: practical lessons to improve patient safety. Arch Pathol Lab Med. 2005;129:1252-1261.

[6] Alenstein PN, Raab SS, Walsh MK. Identification errors involving clinical laboratories: a College of American Pathologists Q-Probes study of patient and specimen identification errors at 120 institutions. Arch Pathol Lab Med. 2006;130:1106-1113

[7] Bhat, Vivek. Tiwari, Manikchandra. Chavan, Preeti. Kelkar, Rohini :Analysis of laboratory sample rejections in the preanalytical stage at an oncology center. Clinica Chimica Acta. 413(15-16):1203-6, 2012 Aug 16.

[8] Snydman, Laura K. Harubin, Beth. Kumar, Sanjaya. Chen, Jack. Lopez, Robert E. Salem, Deeb N: Voluntary electronic reporting of laboratory errors: an analysis of 37,532 laboratory event reports from 30 health care organizations. American Journal of Medical Quality. 27(2):147-53, 2012 Mar-Apr
[9] Hill PM. Mareiniss D. Murphy P. Gardner H. Hsieh YH. Levy F. Kelen GD: Significant reduction of laboratory specimen labeling errors by implementation of an electronic ordering system paired with a bar-code specimen labeling process. Annals of Emergency Medicine. 56(6):630-6, 2010 Dec.

[10] Grimm E. Friedberg RC. Wilkinson DS. AuBuchon JP. Souers RJ. Lehman CM: Blood bank safety practices: mislabeled samples and wrong blood in tube--a Q-Probes analysis of 122 clinical laboratories. Archives of Pathology \& Laboratory Medicine. 134(8):1108-15, 2010 Aug.

[11] Morrison AP. Tanasijevic MJ. Goonan EM. Lobo MM. Bates MM. Lipsitz SR. Bates DW. Melanson SE: Reduction in specimen labeling errors after implementation of a positive patient identification system in phlebotomy. American Journal of Clinical Pathology. 133(6):870-7, 2010 Jun

[12] Yuan S, Astion ML, Schapiro J, Limaye AP. Clinical impact associated with corrected results in clinical microbiology testing. J Clin Microbiol. 2005;43:2188-2193

[13] Astion ML, Krueger-Nielsen S, Davison B, Miller B. Errors and patient outcomes associated with problems in stat chemistry testing. Clin Chem. 2004; 50(suppl 6):A114-A11 abstract.

[14] Nosanchuk JS, Gottmann AW. CUMS and delta checks. A systematic approach to quality control. Am J Clin Pathol. 1974;62:707-712

[15] Astion ML. Putting power into patient safety interventions. Lab Errors Patient Saf. 2005;1:9-11.

[16] Bologna LJ, Mutter M. Life after phlebotomy deployment: reducing major patient and specimen identification errors. J Health Inf Manag. 2002;16:65-70 